\title{
Evaluación de un digestado como fertilizante orgánico
}

\author{
Evaluation of a digestate as organic fertilizer
}

Lili T. Vega C. ${ }^{\text {; }}$ Daniel A. Vega C. ${ }^{2}$; Fernando A. Poveda A. ${ }^{2}$

\section{RESUMEN}

El proceso de biodigestión seca es una alternativa para el manejo de residuos orgánicos sólidos, el cual genera un producto principal denominado biogás y un subproducto llamado digestado. Esta investigación se realizó en Bogotá, Colombia y tuvo como objetivo evaluar el potencial de uso del digestado, identificando alternativas para su utilización en la agricultura. El subproducto obtenido fue sometido a evaluaciones físicas, químicas y microbiológicas, las cuales evidenciaron una estabilidad del material desde las variables estudiadas. Esto permitió concluir que el biodigestado puede ser utilizado en planes de recuperación de suelos y de fertilización como abono y enmienda orgánica no húmica, ya que cumple a cabalidad con las exigencias de la Norma Técnica Colombiana 5167 del 2004. No obstante, se recomienda estabilizar este material a través de procesos de compostaje y así manejar sus contenidos de humedad y microorganismos fitopatógenos.

Palabras clave: abonos orgánicos, residuos orgánicos, efluente, digestado, biodigestión seca.

\section{ABSTRACT}

The dry biodigestion process is an alternative for the management of solid organic waste, which generates a main product called biogas, and a by-product called digestate. Therefore, the present investigation was carried out in Bogotá Colombia and aimed to evaluate the potential of using the digestate, identifying alternatives for its use in agriculture. The by-product obtained was subjected to physical, chemical, and microbiological evaluations, which allowed evidence of a stability of the material from the variables studied. The foregoing allowed us to conclude that the bio digestate can be used in possible soil recovery and fertilization plans as fertilizer and non-humic organic amendment, since it fully complies with the requirements of Colombian Technical Standard 5167 of 2004. However, it is recommends stabilizing this material through composting processes and thus managing its moisture content and possible phytopathogenic microorganisms.

Keywords: organic fertilizers, organic waste, effluent, digestate, dry biodigestion.

\section{Introducción}

De acuerdo con Naciones Unidas, está previsto que la población mundial aumente en más de 1.000 millones en los próximos 13 años. De forma paralela, se ha presentado un crecimiento en el desarrollo tecnológico, en las labores industriales y los servicios, actividades que son en gran medida la base del desarrollo económico de la sociedad. Esta situación ha derivado directamente en la generación de residuos de todo tipo. Se prevé que en los próximos 30 años, la generación de residuos a nivel mundial pasará de 2.010 millones de toneladas registradas en 2016 a 3.400 millones (Kaza, Yao, Bhada-Tata, Woerden y Van, 2018). El manejo de cualquier tipo de residuos se ha convertido en un verdadero problema, para el cual solo se ofrecen soluciones parciales, que finalmente no lo resuelven. Esto ha desencadenado una serie de inconvenientes en los diferentes escenarios, entre los cuales resalta la contaminación del recurso edáfico, el agua y el aire, además de la generación de vectores que repercuten de forma directa en la salud humana.

La actividad humana produce residuos sólidos urbanos, industriales, agrarios, radiactivos, médicos y de laboratorio. Para América Latina, según la ONU, la fracción orgánica representa más de la mitad de los residuos descartados en las ciudades, con un índice que varía bastante de acuerdo con la renta del país (ONU Medio Ambiente, 2017).

\footnotetext{
1 Jardín Botánico de Bogotá José Celestino Mutis, Investigación en energías renovables, Bogotá, Colombia.

2 Corporación Universitaria Minuto de Dios UVD, Bogotá, Colombia.

* Autor por correspondencia: agroecologiavega@gmail.com
} 
La Comisión para la Cooperación Ambiental Canadiense define los residuos orgánicos como cualquier material originado en una planta o animal que puede ser descompuesto por microorganismos, o como los restos, residuos o productos de desecho de cualquier organismo (Commission for Environmental Cooperation - CEC, 2017). En cuanto a los residuos sólidos orgánicos, nacional e internacionalmente desde hace más de una década se vienen planteando soluciones a través de su valoración energética. Tecnologías como la incineración, gasificación, compostaje, sistema de recuperación de gases de vertedero y biodigestión anaerobia han sido evaluadas (Tan, Ho, Hashim, Lee y Taib, 2015). El compostaje y la digestión anaeróbica son opciones de tratamiento de desechos orgánicos que impiden la generación de metano o su liberación a la atmósfera (Kaza et al., 2018).

La biodigestión es un proceso de biodegradación de los residuos sólidos orgánicos, donde una fracción es convertida en biogás. El biogás es un gas combustible que corresponde a una mezcla de dióxido de carbono, metano y trazas de otros gases. El proceso se desarrolla en cuatro fases: hidrólisis, acidogénesis, acetogénesis y metanogénesis, donde el agua es responsable del crecimiento de la población microbiana y también funciona como un agente de taponamiento para todo el sustrato y los reactivos (Kothari, Pandey, Kumar y Tyagi, 2014). El proceso de biodigestión se puede llevar a cabo vía seca y húmeda. La diferencia reside en el porcentaje de sólidos que posee el proceso biológico: en la biodigestión seca el contenido de sólidos va de 20 a $40 \%$, mientras que para la segunda corresponde a un líquido o lodo con un contenido de sólidos del 10 al 15\% (Mery, Herrera, Flores y Bravo, 2018). Los microorganismos involucrados son sensibles a la presencia de oxígeno, y pueden inhibir el proceso. Por lo tanto, es un proceso anaeróbico.

Además de la generación de biogás en el proceso de biodigestión, se obtiene un subproducto denominado efluente o digestado, el cual posee un potencial para ser usado como fertilizante, ya que los nutrientes presentes en el material de entrada (biomasa) permanecen en él y estarán disponibles para los cultivos después del aprovechamiento energético (Kuusik, Pachel, Kuusik y Loigu, 2015). Por lo tanto, el contenido de los nutrientes varía de acuerdo con la biomasa empleada. Las propiedades fisicoquímicas y el potencial de los digestados provenientes de la biodigestión vía húmeda han sido ampliamente investigadas (Kuusik et al., 2015), (Riva et al., 2016), (Bonetta, Bonetta, Ferretti, Fezia, Gilli, y Carraro, 2014), (Søndergaard, Fotidis, Kovalovszki y Angelidaki, 2015) y (Makádi, Tomócsik, y Orosz, 2012). Mediante estos estudios, se encontró que el nitrógeno orgánico contenido en la biomasa se transforma durante el proceso de biodigestión en una forma mineral, como amoníaco, lo que sugiere el uso potencial de digestato como fertilizante en sustitución de fertilizantes minerales (Riva et al., 2016). Además, se reporta que las fracciones orgánicas del digestado pueden contribuir a la rotación de materia orgánica del suelo (SOM), influyendo en las características biológicas, químicas y físicas del suelo como una enmienda del suelo (Makádi et al., 2012).

No obstante, el digestado posee una alta carga mineral y de microorganismos, la cual puede ser foco de contaminación. Durante la etapa termofílica se aumenta la tasa de eliminación de bacterias patógenas y en la mesofílica sola puede no ser adecuada para una correcta higiene, ya que se necesita un tratamiento por separado $\left(70^{\circ} \mathrm{C}, 60 \mathrm{~min}\right.$, tamaño de partícula $<12 \mathrm{~mm}$ ) antes o después de la digestión, especialmente en el caso de subproductos animales (Makádi et al., 2012). Los metales pesados, es decir, parte de la carga mineral, pueden estar presentes, ya que no son alterados por la digestión anaeróbica y pueden concentrarse debido a la reducción de masa durante el proceso (Bonetta et al., 2014). Por otra parte, el mal uso del digestato puede causar molestias para el público (olores) y problemas ambientales como el nitrato y las emisiones de amoníaco en el aire (Riva et al., 2016).

En el Jardín Botánico de Bogotá José Celestino Mutis (JBB) se encuentra instalado un piloto de biodigestión seca con capacidad de $5 \mathrm{~kW}$, el cual es alimentado con residuos de plazas de mercado distritales, corte de césped y estiércol de vaca y de cerdo. Este sistema genera alrededor de 8 toneladas de digestado (residuo sólido) al mes y abre las puertas para identificar el potencial de uso de este residuo en planes de recuperación edáfica y fertilización orgánica de suelos para la producción de alimentos agrícolas.

\section{Materiales y métodos}

La investigación se abordó desde 2 fases: trabajo de campo y trabajo de laboratorio. El 
trabajo de campo se realizó al interior de las instalaciones del Jardín Botánico de Bogotá José Celestino Mutis, empleando el piloto de biodigestión seca. La fase de laboratorio se desarrolló en un laboratorio especializado de pruebas químicas y microbiológicas.

\section{Trabajo de campo}

El piloto de biodigestión seca está conformado por tres reactores tipo Garaje, con tiempo de retención hidráulica de 28 días y una dimensión de digestor de 4,2 $\mathrm{m}$ de largo, 2,5 $\mathrm{m}$ de ancho y $3,2 \mathrm{~m}$ de alto para un volumen total de $33,6 \mathrm{~m}^{3}$. El sistema consta de tres reactores en concreto (Figura 1), con un aislamiento térmico y puertas de marco en acero inoxidable, un cárcamo para la recirculación de lixiviados, un gasómetro y motogenerador. El sistema tiene una capacidad de 15 toneladas de residuos por reactor.

Para dar inicio al proceso, cada reactor se cargó con residuos de plaza de mercado distrital, estiércol de cerdo y vaca, y corte de césped. Estos residuos provienen de las plazas de mercado distritales Santander y Kennedy, frigorífico San Martin y corte de césped del JBB de la ciudad de Bogotá.

Previo al cargue y una vez cualificado el material orgánico, se procedió a la determinación de las variables: peso, volumen y densidad aparente. Para esta actividad, se utilizó balanza digital industrial, capacidad 600 kilos, con plataforma
45 x $60 \mathrm{~cm}$. Además se calculó el volumen por medio de contenedores cilíndricos específicos y se identificó la variable densidad aparente del sustrato utilizado a través de ecuación matemática.

En la fase de campo, se tomó muestra aleatoria representativa de la biomasa de carga del biodigestor. Asimismo, se colectaron $50 \mathrm{~cm}^{3}$ de los lixiviados obtenidos y utilizados dentro del proceso. Para culminar el trabajo de campo, se tomaron muestras del digestado resultante en la fase final del proceso de biodigestión seca. Luego se procederá a la fase de laboratorio y la respectiva caracterización física, química y microbiológica de estos materiales.

Es importante resaltar que se realizaron 3 muestreos de cada parámetro analizado (biomasa, lixiviado y digestado), es decir, una toma de muestra por cada reactor del biodigestor.

\section{Trabajo de laboratorio}

La segunda fase de la investigación se llevó a cabo en el laboratorio especializado de pruebas físicas, químicas y microbiológicas, el cual se encuentra certificado con altos estándares de calidad para el sector agrícola y cuidado del medio ambiente en Colombia. Las pruebas que se contemplaron en el estudio se describen a continuación:

Análisis elemental (CNHSO): para la identificación y cuantificación de nitrógeno $(\mathrm{N})$, carbono (C), hidrógeno y azufre (S) presente en la biomasa.

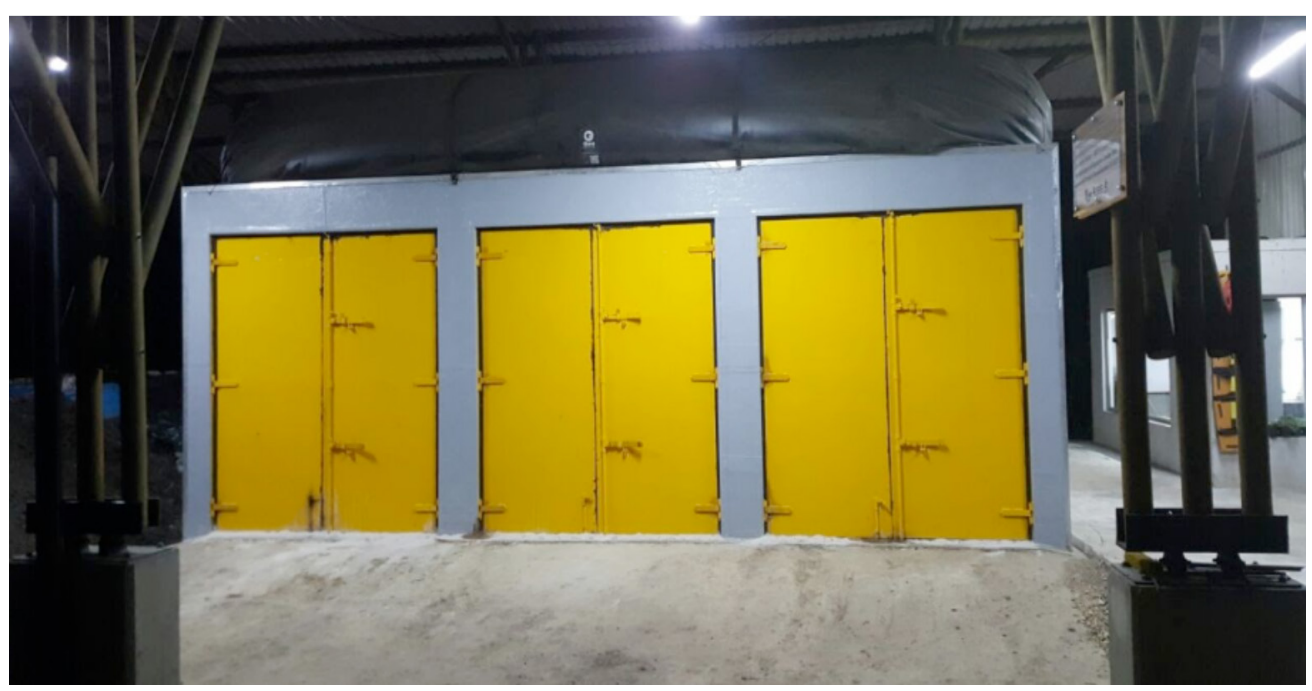

Figura 1. Piloto de biodigestión seca ubicado en Jardín Botánico de Bogotá José Celestino Mutis.

Fuente: Los autores, 2019. 
El equipo utilizado fue un Thermo Flash 2000, con una temperatura en el reactor de $950{ }^{\circ} \mathrm{C}$ y flujo de $\mathrm{He}$ y $\mathrm{O}_{2}$ de $140 \mathrm{ml} / \mathrm{min}$ y $250 \mathrm{ml} / \mathrm{min}$ respectivamente; con una columna de separación PTFE con longitud de $200 \mathrm{~cm}$, OD $6 \mathrm{~mm}$, ID $5 \mathrm{~mm}$ y temperatura de $65^{\circ} \mathrm{C}$. El método utilizado para el análisis de las muestras fue por oxidación por combustión con oxígeno a $1.000{ }^{\circ} \mathrm{C}$.

Metales pesados: este análisis se realizó al lixiviado y al efluente generado del proceso de biodigestión. Se identificaron y cuantificaron los siguientes elementos: cromo $(\mathrm{Cr})$, níquel $(\mathrm{Nq})$ arsénico (As), mercurio $(\mathrm{Hg})$, plomo $(\mathrm{Pb})$ y cadmio (Cd). El objetivo fue determinar posibles fuentes de contaminación por metales pesados en el material evaluado. Este análisis se realizó conforme al Standard Methods for examination of water and wastewater, the AWWA Ed.22/2012 y a través de espectrofotometría de absorción atómica.

Análisis fisicoquímico básico para abonos orgánicos sólidos: se aplicó al digestado con la finalidad de identificar las siguientes variables: $\mathrm{pH}$, conductividad eléctrica, capacidad de intercambio catiónico, nitrógeno total, carbono orgánico, materia orgánica, cenizas, relación carbono nitrógeno $\mathrm{C} / \mathrm{N}$, humedad, densidad y los elementos: azufre total, sodio total, calcio total, magnesio total, potasio y fósforo totales. Asimismo, se identificaron y cuantificaron los siguientes metales pesados: $\mathrm{Cr}$, $\mathrm{Nq}, \mathrm{As}, \mathrm{Hg}, \mathrm{Pb}$ y Cd.

Desde el punto de vista microbiológico se realizó identificación y conteo de bacterias mesófilas aerobias, mohos filamentosos y levaduras, coliformes totales, escherichia coli, detección de salmonella, huevos de helminto, Erwinia sp y Pseudomonas sp. Se aplicó a lixiviado y digestado.

pH: se identificó el pH de los materiales de entrada al biodigestor, y en el afluente resultante del proceso de biodigestión seca. El pH se relaciona de forma directa con la disponibilidad de los elementos minerales para las plantas. Esta variable se medirá con el pH-metro para suelo portátil y de calibración automática, con rangos de medición de 0,00-14,00 pH y una resolución de 0,01 pH.

\section{Análisis estadístico y triangulación de los resultados}

Con la finalidad de reducir significativamente la variabilidad, se trabajó con un diseño de medias repetidas, y se realizaron análisis químicos y microbiológicos a los lixiviados y a la biomasa entrante en el sistema de biodigestión seca, así como al subproducto resultante de este proceso, es decir, al digestado.

Para el análisis estadístico y pruebas de hipótesis se utilizó el Programa PSPP®. Se realizó de forma preliminar una prueba de normalidad (mediante el estadístico no paramétrico Kolmogorov Smirnov), a fin de comprobar la distribución normal de los datos y de esta manera poder sacar conclusiones estadísticamente válidas a partir de los estadísticos descriptivos. Es decir, bajo una hipótesis de trabajo de que la distribución de las variables es diferente a la normal y con una significancia del 5\% para rechazar o no la hipótesis nula. Asimismo, se realizó estadística descriptiva de los datos encontrados en las variables de estudio.

Esta investigación se hizo con la finalidad de identificar el potencial del efluente resultante del proceso de la biodigestión seca y sentar bases científicas que permitan tomar decisiones frente al uso de este material en la agricultura, específicamente para el manejo de suelos y para evitar/mitigar riesgos de contaminación químicos y microbiológicos de suelos, así como efectos negativos sobre la salud.

\section{Resultados}

\section{Biomasa}

La biomasa empleada presentó una variación menor al $10 \%$ en la composición. En promedio el $42 \%$ fue contenido ruminal de vaca y cerdo, $16 \%$ corte de césped y el $42 \%$ restante corresponde a los residuos de plazas de mercado: hortalizas, frutas, verduras, tubérculos, cereales, hierbas aromáticas, alimentos preparados y flores.

En la Tabla 1 se observa la composición en \% de la biomasa empleada y la de diferentes autores.

El análisis elemental de la biomasa arrojó que la composición de la biomasa expresada en

Tabla 1. Composición en \% p/p de la biomasa.

\begin{tabular}{lc}
\hline \multirow{2}{*}{ Composición de biomasa } & Valor $(\% \mathrm{p} / \mathrm{p})$ \\
\cline { 2 - 2 } Nitrógeno total & (Biomasa empleada) \\
Carbono total & 1,58 \\
Hidrógeno total & 46,50 \\
Azufre total & 6,05 \\
\hline
\end{tabular}


su fórmula empírica es $C_{30,7} H_{49} O_{26,1} N_{1}$. Basado en la fórmula química empírica obtenida, la relación $(\mathrm{C}: \mathrm{N})$ del residuo de poda 30,7: 1, este valor es cercano por encima al límite superior recomendado (entre 20 y 30) (Tencio, 2011), por lo tanto, el nitrógeno se va a consumir rápidamente por las bacterias metanogénicas para satisfacer sus necesidades proteicas y no reaccionará más con el contenido restante de carbono. Como resultado, la producción de gas se verá afectada.

- Lixiviado

- Componente químico

- Metales pesados

Los lixiviados fueron analizados desde el punto de vista químico para la detección de $\mathrm{Cr}, \mathrm{Nq}, \mathrm{As}$, $\mathrm{Hg}$, $\mathrm{Pb}$ y $\mathrm{Cd}$ (Figura 2). Para el caso puntual de los tres últimos elementos, se encontró como resultado la no detección de estos metales pesados para los límites de $0,002 \mathrm{mg} / \mathrm{L}$ para mercurio, $0,1 \mathrm{mg} / \mathrm{L}$ para plomo y $0,01 \mathrm{mg} / \mathrm{L}$ para cadmio.

Al realizar prueba de normalidad en la variable de metales pesados, los resultados del p-value del $99 \%$ para $\mathrm{Cr}, \mathrm{Nq}, \mathrm{As}, \mathrm{Pb}, \mathrm{Cd}$ y del $100 \%$ para $\mathrm{Hg}$, permiten afirmar que no hay evidencia estadística suficiente para rechazar la hipótesis de que la distribución de las variables es igual a la normal. Esto quiere decir que los conjuntos de valores asociados a los contenidos de estos metales pesados dentro del lixiviado presentan una distribución normal en los tres reactores.
Los componentes de los lixiviados pueden ser movilizados hacia el suelo, estar en posición de ser absorbidos por las plantas y llegar hacia los mantos acuíferos y afectar los suministros de agua potable (Alonso, Callejón, Jiménez y Ternero, 2002). Autores como Bourg y Loch señalan que la interacción de las sustancias orgánicas solubles con los metales puede ser afectada por el $\mathrm{pH}$ y otras variables (Bourg y Loch, 1995). Por consiguiente, dichos impactos ambientales desde el punto de vista químico están directamente relacionados con la biomasa de alimentación del proceso de biodigestión seca.

Por otro lado, se puede afirmar que los resultados encontrados cumplen con los estándares planteados en la normatividad colombiana asociada a vertimientos líquidos en redes de alcantarillado público y/o cuerpos de agua: Resolución 1074/1997 expedida por la Secretaría Distrital de Ambiente SDA. Resolución 3358 de 1990 de la Corporación Autónoma Regional CAR modificada por la Resolución 166 de 2008 y Resolución 3957 de 2009 SDA. No obstante, para el caso del Cd, se registró un nivel máximo de $0,18 \mathrm{mg} / \mathrm{L}$ de este metal pesado, superando levemente la mencionada norma.

Asimismo, los resultados fisicoquímicos del lixiviado obtenido en el proceso de biodigestión seca mostraron patrones de comportamiento aptos para el uso en agricultura como abono orgánico mineral líquido (Figura 3), al ser estudiado y comparado con la Norma Técnica Colombiana NTC 5167/2011, la cual

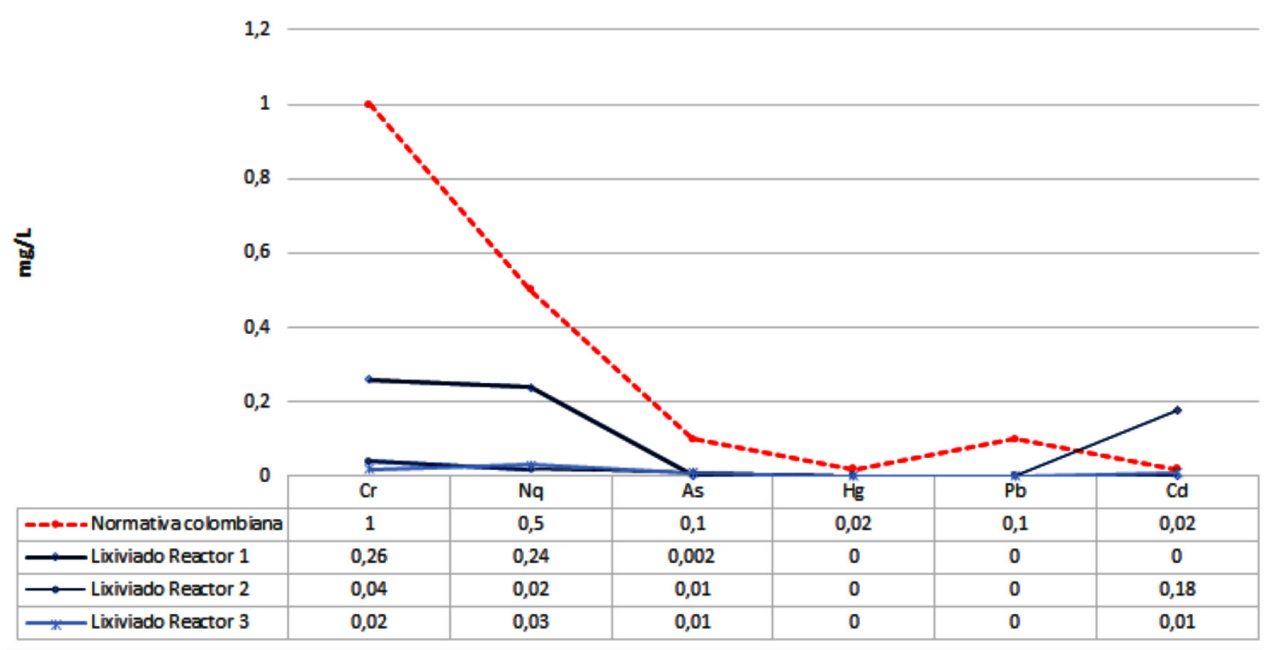

Figura 2. Metales pesados en lixiviados.

Fuente: Los autores, 2019. 


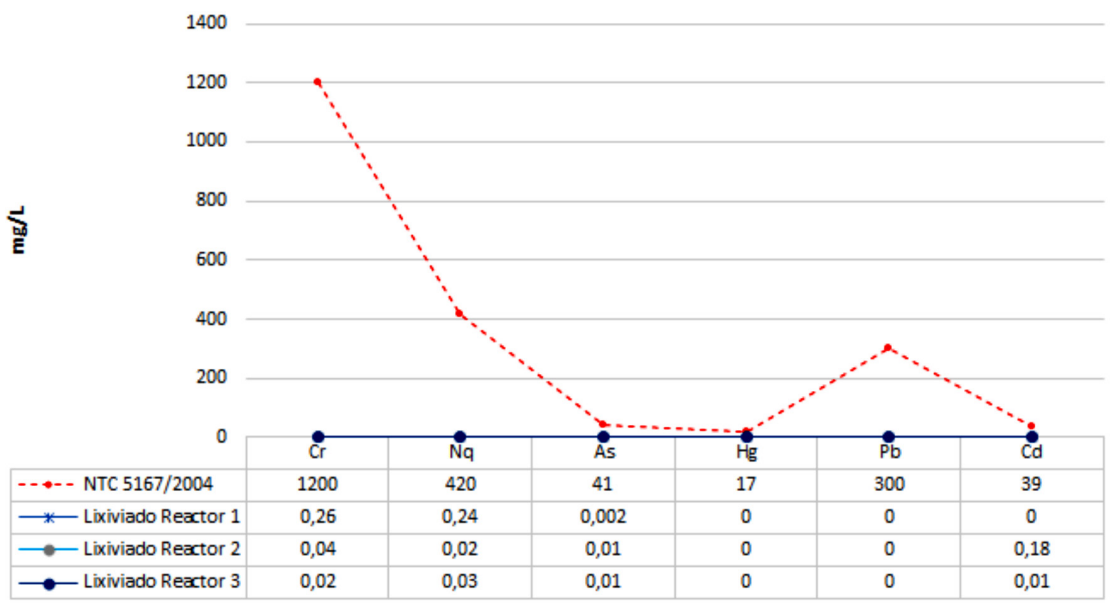

Figura 3. Comparación del lixiviado proveniente de la biodigestión con la normativa vigente.

Fuente: Los autores, 2019.

lo define como "producto líquido obtenido por adición de agua a un abono orgánico, orgánico mineral sólido o mezcla de los anteriores, con posterior extracción al que puede o no, añadírsele un fertilizante mineral" (ICONTEC, 2011). Los valores registrados frente a los metales pesados se encuentran por debajo de los estándares de la normativa colombiana.

\section{Componente microbiológico}

Desde el punto de vista microbiológico, para el caso puntual de la Resolución 3957 de 2009 de la SDA, se estipulan valores de parámetros orgánicos, precisando únicamente el contenido total de coliformes que debe tener un lixiviado que quiera ser descargado en cuerpos de agua. Esta normativa entrega un valor máximo de 20.000 NMP/100 mL y los reportados en este estudio no superaron las $190 \mathrm{UFC} / \mathrm{mL}$.

\section{Digestado}

El pH del digestado obtenido por procesos de digestión seca, en general, varía entre 7,5 y 8,5, para estiércol de ganado y residuos orgánicos sólidos municipales (Schulin, Geiger y Furrer, 1995). El $\mathrm{pH}$ del digestado para esta investigación presentó valores entre ligeramente ácidos y ligeramente alcalinos, con un valor mínimo de 6,28 y un máximo de 7,26. Los aumentos en los valores de $\mathrm{pH}$ en el curso de la biodigestión pueden haber sido causados por la formación de (NH4) 2CO3 (Makádi et al., 2012).

\section{Componente químico Metales pesados}

El contenido de metales pesados en los digestados generalmente se origina a partir de una fuente antropogénica, y estos no son susceptibles a degradación durante el proceso de biodigestión. Por consiguiente, se evidenció la presencia de $\mathrm{Cr}, \mathrm{Nq}, \mathrm{Hg}, \mathrm{Pb}$ y Cd (Figura 4). No obstante, los valores obtenidos están por debajo de los límites permitidos por NTC 5167/2011. Aunado a lo anterior, los metales pesados analizados en el digestado resultante cumplen los estándares planteados por las normativas de la Unión Europea, Alemania y del Reino Unido.

Los resultados encontrados configuran el digestado con un alto potencial para ser utilizado como abono orgánico. No obstante, los metales pesados generan riesgos, ya que pueden potenciar el proceso de bioacumulación en la cadena trófica (Różyło et al., 2017). Por lo tanto, se deben generar estrategias que permitan mitigar la acumulación de trazas de metales pesados en los productos de origen agrícola (Vega y Salamanca, 2016). Se recomienda la separación física (Pérez, 2017) (Smith, Cannon, Woodruff, Solano y Fey, 2013), aunque los metales pesados tienden a desplazarse mayoritariamente a la fase sólida, quedando la fase líquida con un bajo contenido en estos componentes. Para el caso específico, se sugiere realizar una mezcla del digestado (en bajas cantidades) con las pilas de compostaje recién conformadas, con el fin de reducir el contenido de los metales, así como el 


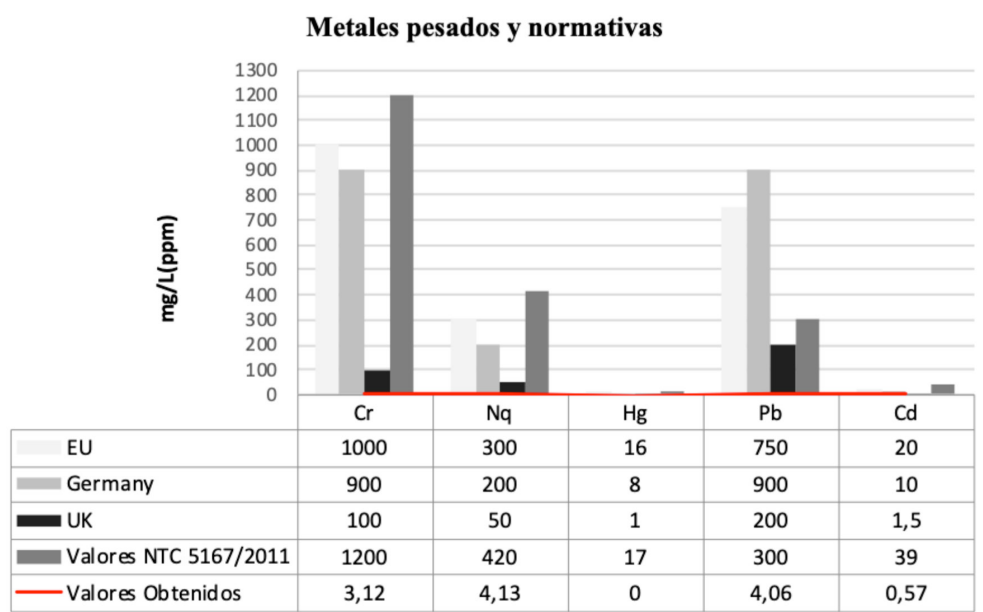

Figura 4. Comparación de contenido de metales obtenidos con reglamentación nacional e internacional en el digestado.

Fuente: Los autores, 2019.

monitoreo constante de los niveles de estos en los abonos orgánicos obtenidos.

\section{Otros elementos}

Dentro de los resultados encontrados se destacan los contenidos de elementos mayores: nitrógeno, fósforo y potasio, así como de menores: calcio, magnesio, sodio y azufre (Figura 5). Dichos elementos son nutrientes minerales para las plantas y "pueden funcionar, además de como constituyente de una estructura orgánica, como activador de una reacción enzimática, transportador de carga, u osmo-regulador" (Valagro, 2004). Por consiguiente, los elementos encontrados en el digestado podrían realizar aportes de nutrientes indispensables para las plantas y que se resumen en crecimiento, desarrollo y rendimiento de los sistemas productivos.

\section{Otras variables analizadas}

Entre las variables químicas más empleadas como indicadores de fertilidad se destacan el $\mathrm{pH}$, la conductividad eléctrica, el contenido de materia orgánica, la capacidad de intercambio catiónico (CIC) y los nutrientes ( $\mathrm{N}$ total, nitratos, amonio, relación C: $\mathrm{N}$, fósforo total y disponible, y potasio) (De la Rosa, 2005; De la Rosa y Sobral, 2008).

El pH encontrado en el digestado arrojó valores óptimos para la producción de alimentos. El rango óptimo de esta variable para la mayoría

\section{Elementos menores y mayores}

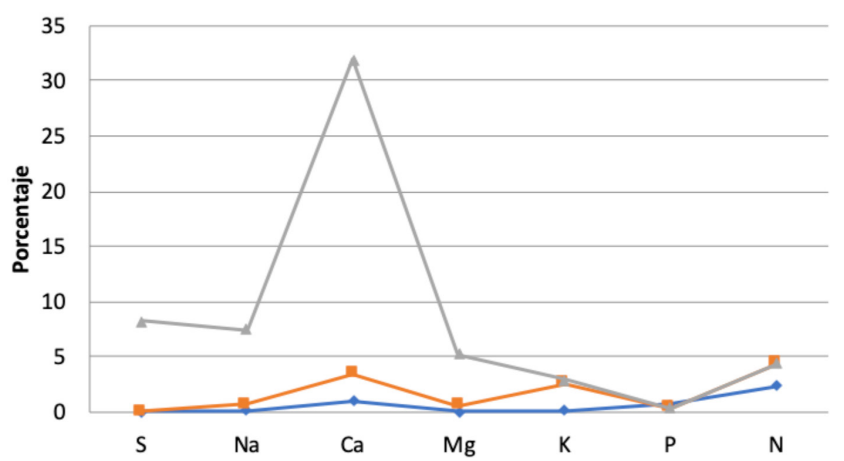

Figura 5. Elementos mayores y menores en digestado. Fuente: Los autores, 2019. 
de los cultivos oscila entre 5,5 y 6,8. Si el pH de un abono-sustrato se encuentra en el rango óptimo, la mayoría de los nutrientes mantiene su máximo nivel de solubilidad. Por debajo de este rango, pueden presentarse deficiencias de elementos mayores; mientras que, por encima, puede disminuir la solubilidad de algunos elementos menores. (Barbaro, Karlanian y Mata, 2018).

La conductividad eléctrica (CE) provee una medida de la cantidad de fertilizante que se encuentra disponible en el medio para el crecimiento de las plantas o indica si existe acumulación de sales (Torres, Camberato, López y Mickelbart, 2010), y para este caso puntual y las demás variables analizadas, se observaron niveles óptimos al ser comparados con los parámetros fijados por la NTC 5167/2011 Productos para la Industria Agrícola (Tabla 2).

\section{Componente microbiológico}

Para el componente microbiológico del digestado (Tabla 3) se encontraron niveles aceptables por la normativa colombiana. No obstante, se registraron valores elevados de fitopatógenos (Tabla 3), los cuales pasan parte de su ciclo de vida en las plantas que les sirven de hospedante, y otra parte en el suelo o en los residuos vegetales depositados en este sustrato (Agrios, 2005). Por consiguiente, es recomendable que al finalizar el proceso de producción de gas, el digestado resultante sea sometido a procesos de estabilización de la materia orgánica, como compostaje, el cual ha demostrado ser un método eficaz para el control de organismos patógenos: Salmonella, Shiguella, Klebsiella, Pseudomonas y Escherichia coli. Estos patógenos pueden presentarse en la entrada del proceso, pero en la etapa termófila son erradicados (Escudero de Fonseca y Arias, 2012).

\section{Conclusiones}

Hablar de lixiviados, los riesgos asociados a la contaminación química-biológica y sus efectos sobre el medio ambiente de manera generalizada, puede generar confusión al momento de su entendimiento y tratamiento. Se debe tener presente que existe una relación directa entre el tipo de residuo y los contaminantes que puede generar. Por lo tanto, conocer la procedencia de los

Tabla 2. Composición en \% p/p de la biomasa.

\begin{tabular}{lcccc}
\hline Variable & Unidad & Reactor 1 & Reactor 2 & Reactor 3 \\
\hline $\mathrm{pH}$ & Unidades & 7,26 & 6,28 & 6,52 \\
$\mathrm{CE}$ & $\mathrm{dS} / \mathrm{m}$ & 0,87 & 1,14 & 12,9 \\
Relación C/N & $\%$ & 6,71 & 3,33 & 1,26 \\
Cenizas & $\%$ & 1,35 & 13,8 & 0,61 \\
Humedad & $\%$ & 74,12 & 72,9 & 78,6 \\
Carbono orgánico & $\%$ & 15,9 & 14,6 & 5,48 \\
Materia orgánica & $\%$ & 27,5 & 25,2 & 9,44 \\
Densidad & $\mathrm{g} / \mathrm{cm} 3$ & 0,13 & 0,11 & 0,11 \\
CIC & $\mathrm{meq*100-1}$ & 63,4 & 96,3 & 96,3 \\
\hline
\end{tabular}

Tabla 3. Composición en \% p/p de la biomasa.

\begin{tabular}{llrrr}
\hline \multicolumn{1}{c}{ Variable } & \multicolumn{1}{c}{ Unidad } & Reactor 1 & Reactor 2 & Reactor 3 \\
\hline E. Coli & UFC/ml & $<1$ & $<1$ & 33 \\
Salmonela & g de materia fresca. & Ausente & Ausente & Ausente \\
Coliformes & UFC/ml & 1600 & 79 & 76 \\
Huevos de helminto viables & \# huevo/g de muestra & 0 & 1 & 0 \\
Fitopatógenos & UFC/ml & 27000 & 28000 & 27000 \\
Pseudomonas sp. & UFC/ml & 0 & 0 & 0 \\
Erwinia sp. & N/A & 0 & 0 & 0 \\
\hline
\end{tabular}


residuos, sus características, así como los factores que intervienen en la obtención de lixiviados, es de suma importancia para poder sentar bases que permitan tener mayor claridad sobre su tratamiento o uso potencial en procesos de biodigestión seca.

El proceso de biodigestión seca puede ser considerado como una estrategia de ciclo cerrado para el tratamiento de residuos sólidos orgánicos, ya que al ser manejado de manera técnica permite, además de la generación de biogás, la obtención de subproductos con altos potenciales de uso para la fertilización de cultivos y la recuperación de suelos. Esto gracias a los aportes del biodigestado en términos químicos, físicos y microbiológicos.

Es recomendable iniciar procesos de compostaje a los digestados obtenidos en la biodigestión seca, con la finalidad de someter este subproducto a procesos de estabilización de la materia orgánica, que permitan en gran medida eliminar agentes patógenos y fitopatógenos presentes en esta y que podrían causar efectos negativos para la salud de las personas y los sistemas productivos agrícolas. De forma simultánea, este proceso contribuiría a mejorar de manera significativa los contenidos de humedad del subproducto obtenido.

Es de suma importancia continuar investigando el uso de este subproducto directamente en sistemas productivos agrícolas, que permita determinar dosis y épocas de aplicación, así como los parámetros asociados a los beneficios económicos, ecológicos y sociales por la utilización de este tipo de biotecnología en la producción de alimentos.

\section{Literatura Citada}

Agrios, G.N.

2005. Fitopatología, 2da edición. Limusa, México. 952 p.

Alonso Álvarez, E.; Callejón Mochón, M.; Jiménez Sánchez,

J.; Ternero Rodríguez, M.

2002. Heavy metal extractable forms in sludge from wastewater treatment plants. Chemosphere, 47: 765-775.

Barbaro, L.; Karlanian, M.; Mata, D.

2018. Importancia del pH y la Conductividad Eléctrica (ce) en los sustratos para las plantas. Presidencia de la Nación. Ministerio de Agricultura, Ganadería y Pesca. Argentina. $11 \mathrm{p}$.

Bonetta, S.; Bonetta S.; Ferretti, E.; Fezia, G.; Gilli, G.; Carraro, E. 2014. Agricultural Reuse of the Digestate from Anaerobic CoDigestion of Organic Waste: Microbiological Contamination, Metal Hazards and Fertilizing Performance. Water Air Soil Pollut, 225(8): 2046. DOI: 10.1007/s11270-014-2046-2

Bourg, A.; Loch, G.

1995. Mobilization of Heavy Metals as Affected by $\mathrm{pH}$ and Redox Conditions. En: Salomons, W.; Stigliani, W.N. (eds.).Biogeodynamics of Pollutants in Soils and Sediments. Springer Verlag, Berlin, Alemania. pp. 87-102.

CEC.

2017. Characterization and Management of Organic Waste in North America. Foundational Report. Commission for Environmental Cooperation. Montreal, Canada. 260 p.

De la Rosa, D.

2005. Soil quality and monitoring based on land evaluation. Land Degradation \& Development, 16: 551-559.

De la Rosa, D.; Sobral, R.

2008. Soil quality and methods for its assessment. En: Braimoh A.K.; Vlek P.L.G. (eds) Land Use and Soil Resources. Springer, Dordrecht. pp. 167-200.

Escudero de Fonseca, A.; Arias, C.

2012. Los microorganismos en los abonos orgánicos a partir de podas en la Universidad del Norte, Colombia. Revista Internacional de Contaminación Ambiental. 28 (1): 67-75.
ICONTEC.

2011. Norma Técnica Colombiana NTC 5167/2011. Productos para la Industria Agrícola. Productos Orgánicos usados como Abonos o Fertilizantes y Enmiendas o Acondicionadores de Suelo. Instituto Colombiano de Normas Técnicas y Certificación. Bogotá, Colombia. 40 p.

Kaza, S., Yao, L., Bhada-Tata, P.; Woerden, F. Van.

2018. WHAT A WASTE 2.0 A Global Snapshot of Solid Waste Management to 2050. World Bank. Washington, DC, US. 295 p.

Kothari, R.; Pandey, A. K.; Kumar, S.; Tyagi, V. V.; Tyagi, S. K. 2014. Different aspects of dry anaerobic digestion for bioenergy: An overview. Renewable and Sustainable Energy Reviews, 39: 174-195.

Kuusik, A.; Pachel, K.; Kuusik, A.; Loigu, E. 2015. Possible agricultural use of digestate. Proceedings of the Estonian Academy of Sciences, 64: 64-74.

Makádi, M.; Tomócsik, A.; Orosz, V.

2012. Digestate: A New Nutrient Source - Review. En: Kumar, S. (trac.). Biogas. IntechOpen. pp. 295-310.

Mery, C.S.; Herrera Urrutia, J.; Flores, V.C.; Bravo Ibáñez, M. 2018. Comparación entre generación de biogás por medio de digestión anaerobia seca y húmeda, de residuos orgánicos provenientes de ferias libres. $3^{\circ}$ Congreso Iberoamericano de cambio climático. 8-10/may/ 2018. Buenos Aires, Argentina. 7 p.

ONU Medio Ambiente.

2017. Aumenta la generación de residuos en América Latina y el Caribe mientras 145.000 toneladas aún se disponen de forma inadecuada cada día. Disponible en: https://www. unenvironment.org/es/news-and-stories/reportajes/aumentala-generacion-de-residuos-en-america-latina-y-el-caribe Consultado: 13/nov/2018.

Riva, C.; Orzi, V.; Carozzi, M.; Acutis, M.; Boccasile, G.; Lonati, S.; Adani, F.

2016. Short-term experiments in using digestate products as substitutes for mineral $(\mathrm{N})$ fertilizer: Agronomic 
performance, odours, and ammonia emission impacts. Sci. Total Environ., 547: 206-214.

Różyło, K.; Świeca, M.; Gawlik-Dziki, U.; Andruszczak, S.; Kwiecińska-Poppe, E.; Kraska, P.

2017. Phytochemical properties and heavy metal accumulation in wheat grain after three years' fertilization with biogas digestate and mineral waste. Agricultural and Food Science, 26(3): 148-159.

Schulin, R.; Geiger, G.; Furrer, G.

1995. Heavy Metal Retention by Soil Organic Matter under Changing Environmental Conditions. En: Salomons, W.; Stigliani, W. (Eds.) Biogeodynamics of Pollutants in Soils and Sediments Berlin, Heidelberg: Springer Berlin Heidelberg. Pp. 53-85.

Secretaría Distrital de Ambiente.

2009. RESOLUCIÓN 3957 DE 2009. Norma técnica para el control y manejo de los vertimientos realizados a la red de alcantarillado público en el Distrito Capital. Recuperado del sitio de Internet de http://www.ambientebogota.gov.co/: http://www.alcaldiabogota.gov.co/sisjurMantenimiento/ normas/Norma1.jsp?i=37051.
Søndergaard, M.M.; Fotidis, I.A.; Kovalovszki, A.; Angelidaki, I. 2015. Anaerobic Co-digestion of Agricultural Byproducts with Manure for Enhanced Biogas Production. Energy \& Fuels, 29 (12): 8088-8094

Tan, S.T.; Ho, W.S., Hashim, H.; Lee, C.T.; Taib, M.R.; Ho, C.S. 2015. Energy, economic and environmental (3E) analysis of waste-to-energy (WTE) strategies for municipal solid waste (MSW) management in Malaysia. Energy Conversion and Management, 102: 111-120.

Torres, A.; Camberato, D.; López, R.; Mickelbart, M. 2010. Producción Comercial de Cultivos Bajo Invernadero y Vivero. Medición de pH y Conductividad Eléctrica en Sustratos. Departamento de Horticultura y Arquitectura de Áreas Verdes, Purdue University. Indiana, US. 6 p.

Valagro, SpA.

2004. Los microelementos en la nutrición vegetal. Valagro, SpA. Lanciano, Ch, Italia. 71 p.

Smith, D.B.; Cannon, W.F.; Woodruff, G.; Solano, F.; Kilburn, J.E.; Fey, D.L.

2013. Geochemical and mineralogical data for soils of the conterminous United States. USGS Data Series 80. 19 p. 